\title{
Look Closely: Lessons Prof. Gérard T. Simon Might Have Taught
}

\author{
David N. Howell, MD, PhD \\ Department of Pathology, Duke University and Veterans Affairs Medical Centers, Durham, NC, USA
}

This symposium honors Prof. Gérard T. Simon, a respected colleague in the field of ultrastructural pathology. Though he distinguished himself during the several decades of his career as an outstanding diagnostician, laboratorian, and investigator, he is perhaps best known as an educator and evangelist for the importance of electron microscopy, particularly for trainees. It is no surprise that the annual young investigator awards offered by the Microscopical Society of Canada bear his name; the fact that awardees can be either graduate students or technologists is in harmony with Dr. Simon's clear passion for both the biological and the technical facets of his work.

This presentation is a compendium of diagnostic tips that I have offered to trainees and other coworkers during a long career as a renal pathologist and teacher. It describes diagnostic situations in which the observer is advised to look more closely than usual at the question at hand, not so much in the sense of employing higher magnification (though electron microscopy is certainly the ideal tool for doing that) as in applying additional thought to the problem and perhaps viewing it from a novel angle. Though I never had the pleasure of meeting Prof. Simon, I suspect from his writings that his approach was similar.

A closer look is often warranted when one receives a suboptimal diagnostic specimen. Shortcomings can include limited quantity as well as poor quality (for reasons such as inappropriate or inadequate fixation and careless, rough manipulation). A natural tendency with such specimens is to give them abbreviated consideration based on the premise that they are unlikely to provide useful information, a reflex that should be avoided. Electron microscopy is an ideal method for examining small specimens (which are necessary as well as often sufficient for ultrastructural analysis), and in many cases is surprisingly forgiving of specimen mishandling. It is frequently possible to establish an ultrastructural diagnosis on a renal biopsy with a single glomerulus, as many causes of glomerular dysfunction (e.g., immune complex and other deposits, injury to epithelial or endothelial cells, basement membrane defects) often have a diffuse ultrastructural distribution. (But unfortunately not always; see below.)

Extra scrutiny is also advisable in situations where diagnosticians or clinical colleagues have a preconceived notion of what the diagnosis is going to be. It is easy to be lulled into seeing what one expects to see, either on the basis of clinical information or of initial studies performed on a sample. Diagnostic tests are often tailored to achieve the anticipated diagnosis, particularly when analytespecific reagents like antibodies or nucleic acid probes are employed; if one expects to find cytomegalovirus (CMV) infection in a histologic specimen, one typically stains it with an anti-CMV antibody, and risks missing infections with other viruses. Electron microscopy circumvents this problem by providing what Prof. Hans Gelderblom has described as an "open view": an unbiased physical representation of what is present that does not depend directly on biochemical makeup.

In a similar manner, diagnosticians often encounter the pitfall of detecting one pathologic process and considering the job finished. Patients, particularly the severely or chronically ill, often have more than one thing wrong, and specimens sometimes hold diagnostic keys to multiple maladies. To extend the example above, a positive stain for CMV does not preclude the concomitant presence of another virus 
(or some other pathogen or non-infectious abnormality). I have encountered at least one diagnostic tissue sample (from the autopsy of an immunocompromised patient) infected with both CMV and herpes simplex virus; the coinfection was initially suspected based on disparate forms of cytopathic effect in a simple hematoxylin-and-eosin stain. In the same way that the "open view" of electron microscopy can help avoid the bias of diagnostic preconception, it can help insure that multiple disease processes are not overlooked. (In a sense, the second of these pitfalls is a special case of the first in which finding the initial abnormality biases the observer against looking for more.)

At the opposite end of the spectrum, increased vigilance is recommended in situations where the observer has little or no notion of what is wrong with a patient or specimen, even after considerable preliminary examination. On the surface, this would seem obvious, yet there is occasionally a temptation to throw in the towel early in such cases and assume that the problem is insoluble or that the pathologic process afflicting the patient is not represented in the tissue available for study. The vast and growing armamentarium of special stains and molecular tests currently available is of little use (or at least prohibitively expensive) if one has no idea which ones to employ, sometimes inducing "diagnostic paralysis" in young pathologists trained to depend on such methods. For the reasons discussed above, electron microscopy often provides a way around this impasse.

Another shortcut with potential for causing error is to concentrate one's attention in areas of obvious pathologic alteration to the exclusion of portions of the specimen that initially appear normal. The latter areas sometimes harbor important (often ultrastructural) diagnostic clues. In a variety of glomerular disorders that present clinically with proteinuria and/or hematuria (e.g., minimal change disease, thin basement membrane nephropathy, early stages of any number of glomerulopathies), glomerular changes may be negligible at the light microscopic level. In some cases, the entire biopsy may appear relatively normal by light microscopy; in others, secondary tubulointerstitial changes (epithelial vacuolization, tubular erythrocyte casts, interstitial foam cells) may be the only noticeable alterations. It's important to look at the whole specimen with care, even in places where you don't think you should be looking.

Though looking closely has been used in this presentation in the sense of looking (and thinking) carefully rather than at high magnification (much of the best close looking is done at low-to-medium power), it should be noted in closing that careful scrutiny of a specimen for focal or minute details is often essential. Small, localized deposits of granular, fibrillar, or microtubular material are often harbingers of disorders such as immune complex glomerulonephritis, amyloidosis, fibrillary glomerulonephritis, or cryoglobulinemic glomerulonephritis in their earliest stages. Female carriers of X-linked heritable disorders such as Alport syndrome and Fabry disease may have subtle, focal glomerular ultrastructural alterations (capillary loop basement membrane irregularities in the former, lamellated intracellular inclusions termed "zebra bodies" in the latter). Tubuloreticular inclusions, minute structures usually seen in capillary endothelial cells, are often an early warning sign for systemic lupus erythematosus or human immunodeficiency virus infection.

In short, the safest approach is to examine every specimen with care. In an address on the occasion of the $25^{\text {th }}$ anniversary of the Microscopical Society of Canada, Prof. Simon closed with a quotation from Jean Monnet, a founder of the European Union: 'L'important ce n'est pas ce que l'on a créé mais ce qui en est advenu." (What is important is not what one founded but what came out of it.) In a more general sense, I think this means that what one does is less important that what one teaches others. If any of my diagnostic philosophy rings true to you, by all means use it, but also pass it on! 